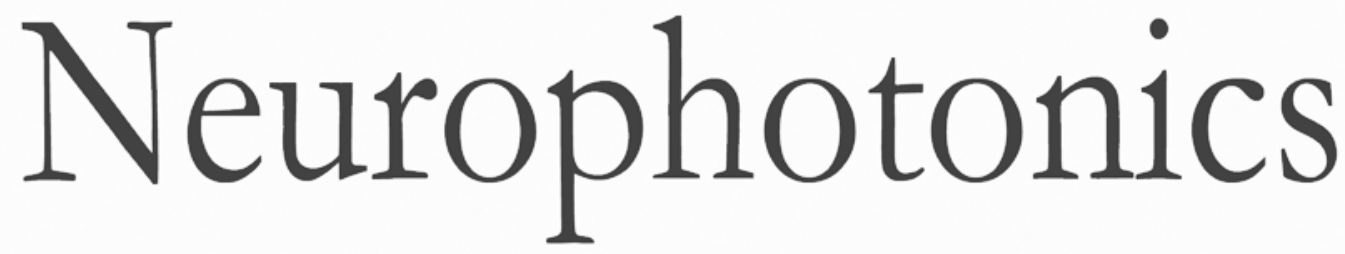

\title{
Association between hemodynamic activity and motor performance in six-month-old full-term and preterm infants: a functional near-infrared spectroscopy study
}

Suelen Rosa de Oliveira

Ana Carolina Cabral de Paula Machado

Jonas Jardim de Paula

Paulo Henrique Paiva de Moraes

Maria Juliana Silvério Nahin

Lívia de Castro Magalhães

Sergio L. Novi

Rickson C. Mesquita

Débora Marques de Miranda

Maria Cândida Ferrarez Bouzada 


\title{
Association between hemodynamic activity and motor performance in six-month-old full-term and preterm infants: a functional near-infrared spectroscopy study
}

\author{
Suelen Rosa de Oliveira, ${ }^{\mathrm{a}, \star}$ Ana Carolina Cabral de Paula Machado, ${ }^{\mathrm{a}}$ Jonas Jardim de Paula, ${ }^{\mathrm{a}}$ \\ Paulo Henrique Paiva de Moraes, ${ }^{a}$ Maria Juliana Silvério Nahin, ${ }^{a}$ Lívia de Castro Magalhães, ${ }^{b}$ Sergio L. Novi, ${ }^{c}$ \\ Rickson C. Mesquita, ${ }^{\mathrm{C}}$ Débora Marques de Miranda, ${ }^{\mathrm{a}}$ and Maria Cândida Ferrarez Bouzada ${ }^{\mathrm{a}}$ \\ aUniversidade Federal de Minas Gerais, School of Medicine, Belo Horizonte, Brazil \\ bUniversidade Federal de Minas Gerais, School of Physical Education, Physiotherapy, and Occupational Therapy, Belo Horizonte, Brazil \\ 'University of Campinas, Institute of Physics, Campinas, Brazil
}

\begin{abstract}
This study aimed to assess task-induced activation in motor cortex and its association with motor performance in full-term and preterm born infants at six months old. A cross-sectional study of 73 sixmonth-old infants was conducted (35 full-term and 38 preterm infants). Motor performance was assessed using the Bayley Scales of Infant Development third edition-Bayley-III. Brain hemodynamic activity during motor task was measured by functional near-infrared spectroscopy (fNIRS). Motor performance was similar in full-term and preterm infants. However, differences in hemodynamic response were identified. Full terms showed a more homogeneous unilateral and contralateral activated area, whereas in preterm-born the activation response was predominantly bilateral. The full-term group also exhibited a shorter latency for the hemodynamic response than the preterm group. Hemodynamic activity in the left sensorimotor region was positively associated with motor performance measured by Bayley-III. The results highlight the adequacy of fNIRS to assess differences in task-induced activation in sensorimotor cortex between groups. The association between motor performance and the hemodynamic activity require further investigation and suggest that fNIRS can become a suitable auxiliary tool to investigate aspects of neural basis on early development of motor abilities. () 2017 Society of Photo-Optical Instrumentation Engineers (SPIE) [DOI: 10.1117/1.NPh.5.1.011016]
\end{abstract}

Keywords: near-infrared spectroscopy; premature infant; sensorimotor cortex; infant development; functional activation. Paper 17069SSR received Mar. 31, 2017; accepted for publication Sep. 18, 2017; published online Oct. 12, 2017.

\section{Introduction}

Premature infants are at high risk for neurodevelopmental impairment even in the absence of any identifiable event involving possible brain injury. ${ }^{1}$ Motor development is one of the most affected areas by premature birth, potentially causing a substantial number of limitations in later life. ${ }^{2}$ Advanced motor skills are determined by a brain and neuromuscular maturation, ${ }^{3}$ in addition to factors such as physical growth, caregiving practices, opportunities for practicing emerging skills, and favorable emotional, social, and environmental conditions. ${ }^{4}$

Since human neurodevelopment is a dynamic interplay between biology and environment, and it can be changed by interventions in one of these features, it is interesting to have accurate instruments to identify early disabilities in infants at risk for neurodevelopment delays, as in prematurely born infants. ${ }^{5}$ This has aroused interest in the understanding of how brain activity relates to the manifestation of a particular behavior or action, especially at early stages of neurodevelopment. Improving our understanding of these factors could be helpful in ameliorating our ability to identify and measure impairments in the course of early childhood development.

*Address all correspondence to: Suelen Rosa de Oliveira, E-mail: sufmg@ yahoo.com.br
The motor and sensory areas appear particularly sensitive to events in late pregnancy since fetal development is rapid between 20 and 37 weeks of gestational age (GA), ${ }^{6}$ and many of the sensorimotor networks are initially established during the second half of gestation. ${ }^{7}$ Motor and sensory areas are one of the most common sites of perinatal brain damage probably due to their high sensibility aforementioned. ${ }^{6}$

In this context, functional near-infrared spectroscopy (fNIRS) appears to be a promising technique for child development studies offering greater safety and operability. ${ }^{8}$ The principles of fNIRS are based on the fact that neural activity is accompanied by changes in oxygenation and also in cerebral blood flow and blood volume. ${ }^{9}$ In fNIRS, changes in oxy-hemoglobin (HbO), deoxy-hemoglobin (HbR), and total hemoglobin concentrations are estimated by detecting near-infrared light ( 700 to $900 \mathrm{~nm}$ ) scattered from tissue, which allows one to evaluate quantitatively and qualitatively hemodynamic changes in microvasculature due to brain activation.

Considering the importance for improving early motor skills measures, especially in preterm infants, we compared, in a transversal study, six-month-old full-term and preterm infants performing a motor task registering their cortical hemodynamic 
responses using fNIRS and its association with motor performance results.

\section{Materials and Methods}

\subsection{Study Design}

This was a cross-sectional study performed between September 2013 and April 2015. Motor performance and cortical activity assessments were evaluated during a single session, at six months of age. For preterm infants, we considered their corrected age. All parents gave their written informed consent, and the study was approved by the Human Ethics Committee of the Federal University of Minas Gerais (UFMG), Brazil. The study was in accordance with the Helsinki Declaration.

\subsection{Participants}

Seventy-three infants participated in this study were allocated into two groups: full term and preterm. Both groups were born between 2013 and 2014 at the maternity unit of the UFMG University's Hospital. The full-term group included 35 infants born with GA > 37 weeks and with no known medical diagnoses or delays. The preterm group included 38 infants born at the same maternity hospital with $\mathrm{GA}<34$ weeks. They were recruited from the institution's follow-up program for highrisk children. Infants with severe sensory impairments (blindness, deafness), cerebral palsy, genetic syndromes or any malformation, congenital heart defects, Apgar score less than 7 at $5 \mathrm{~min}$, intraventricular hemorrhage grade 3 or 4 , and periventricular leukomalacia, were excluded. All children underwent a cranial ultrasound to exclude morphological brain abnormalities.

We obtained fNIRS data from 51 infants (26 full-term and 25 preterm). The remaining 22 infants were tested but not included in the sample due to high motion artifacts caused by: (i) crying during the experiment $(n=16)$; (ii) large head movements $(n=4)$; and (iii) stimulus refusal $(n=2)$. All 73 infants' motor development was assessed before the fNIRS experiment.

\subsection{Procedures}

\subsubsection{Characterization of the studied population}

Information such as infant birth data, socioeconomic status (SES), and neonatal morbidities were collected from medical records. SES was assessed by the Brazilian Economic Classification Criterion (CCEB) ${ }^{10}$ The CCEB is an economic segmentation tool that uses household characteristics to classify population according to average monthly income. CCEB results were categorized into two groups considering monthly income values: "monthly income $>\$ 455$ " and "monthly income $<\$ 455$."

\subsubsection{Motor performance assessment}

Motor performance was assessed using the motor scale of the Bayley Scales of Infant Development (BSID), composed by fine and gross motor subtests. ${ }^{11}$ The BSID is the most commonly used instrument to assess early development, both in clinical practice and research settings. The last edition, Bayley-III, includes five distinct scales to assess cognitive, receptive/ expressive communication, social-emotional, adaptive behavior, and fine/gross motor development. ${ }^{11}$ The Bayley-III assesses developmental functioning through items administered in a structured play format. The motor composite score was calculated by using test norms $($ mean $=100 ; \mathrm{SD}=15) .{ }^{11}$

The same researcher conducted all motor development assessments. Infants who scored in the delayed range on the Bayley-III were included in the study and readily referred to pediatric follow-up at the institution where the study was developed.

\subsubsection{Functional experimental protocol}

We performed a motor functional activation experiment with a block paradigm. The motor stimulus consisted of a vibration produced by a microdirect current motor (coreless motor $9321,6 \times 10 \mathrm{~mm}, 6200 \mathrm{R} / \mathrm{min}$ or $103.3 \mathrm{~Hz}$ ) that was held against the infant's right hand.

The motor stimulus was presented for $8 \mathrm{~s}$ of continuous stimulation followed by a $20 \mathrm{~s}$ rest period between trials [Fig. 1(a)]. In total, we performed eight trials of the task. Trials affected by motion artifacts were discarded. Each infant contributed with at least four blocks of motor data. The infants were seated on their parent's lap during the trial [Fig. 1(b)]. To reduce motion artifact during the experiment, they watched a colorful silent video throughout the data acquisition protocol.

\subsubsection{Near-infrared spectroscopy acquisition and analysis}

Hemodynamic activity in response to functional activation of motor areas was assessed by a commercial continuous-wave near-infrared spectroscopy (NIRS) equipment (NIRScout Tandem 1616, NIRx Medical Technologies, Glen Head). The system employs 32 sources (each source has 2 LED emitters at 760 and $850 \mathrm{~nm}$ ) and 32 detectors. The optodes were bilaterally positioned in the frontal, parietal, temporal, and occipital areas, according to the international 10-20 system coordinates ${ }^{12}$ [Fig. 1(c)]. In this study, we used 30 sources and 28 detectors, providing 84 different source-detector combinations (channels) with source-detector distances ranging from 1.9 to $3.5 \mathrm{~cm}$. From the 84 channels, 38 of those were positioned in the frontal lobe and 16 channels were located in the parietal lobe. The temporal and occipital lobes contained 22 and 8 channels, respectively. More importantly, the probe had 10 channels per hemisphere positioned in the sensorimotor cortex.

For fNIRS data analysis, we used homemade scripts based on HomER 2 functions $^{13}$ in MATLAB (Mathworks, Inc., Natick, Massachusetts). First, channels with low signal-to-noise ratio (SNR) were discarded from analysis. By analyzing the power spectrum, we empirically found that for infants' data and our commercial system, channels with $\mathrm{SNR}<8$ contain no significant hemodynamic information when compared to noise. Therefore, a threshold of 8 for SNR was employed in channel removal. With the remaining channels, motion artifacts were visually inspected and manually removed from the data. Detected light intensity for each channel was bandpass filtered between 0.003 and $0.5 \mathrm{~Hz}$ to eliminate DC trends and physiological interference (mostly heartbeat, breathing) and high-frequency artifacts. Then, time series of hemodynamic concentrations were computed for each channel by using the modified Beer-Lambert's law. ${ }^{14}$

For each subject, the hemodynamic response function (HRF) of each channel was independently obtained by averaging all trials from $5 \mathrm{~s}$ before the start of stimulation until $20 \mathrm{~s}$ after the beginning of the stimulation. For each channel, we compared the amplitude of the time points during the period of stimulation to the amplitude of the time points during the 

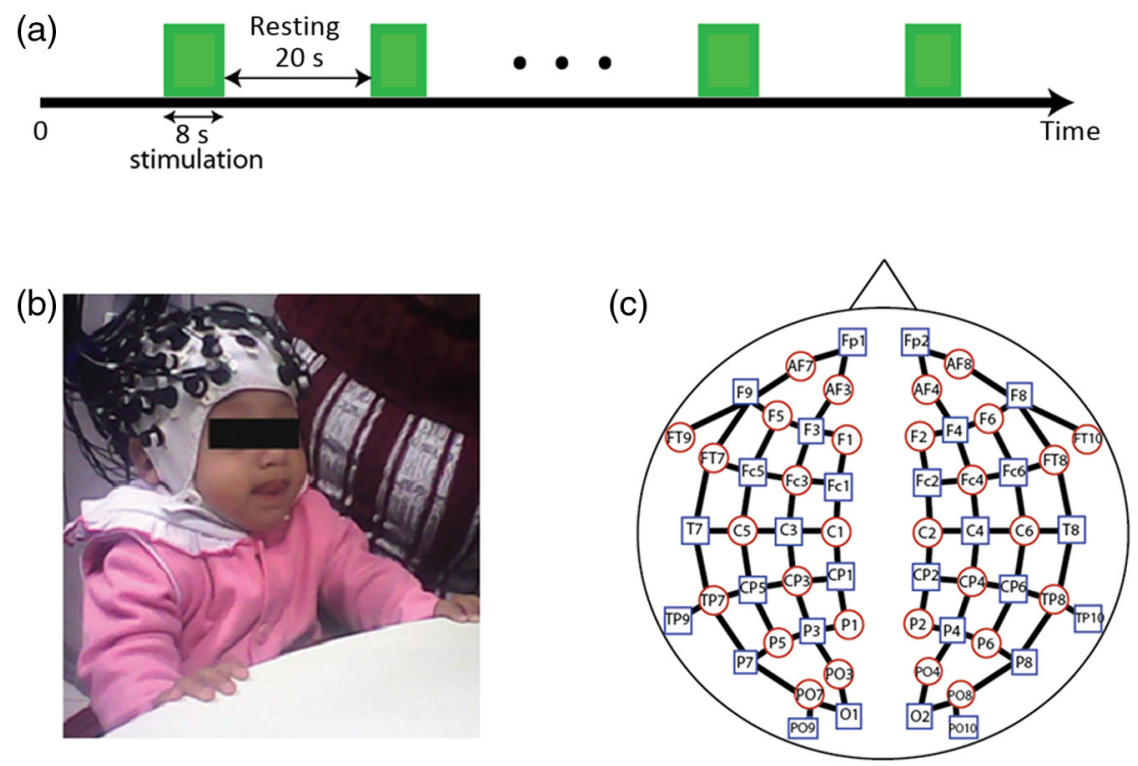

Fig. 1 (a) Functional experimental protocol consisting of 8 trials of $8 s$ of motor stimulation followed by $20 \mathrm{~s}$ of resting. (b) Photo of a six-month-old infant in its mother's lap during fNIRS acquisition. (c) Topographic (two-dimensional) figure showing the arrangement of all sources (red circles) and detectors (blue squares) according to the international 10-20 system coordinates. The source-detector combinations (channels) used in this study are represented by the black solid lines.

baseline (i.e., prior to stimulation). The activated channels were defined as the channels that showed a significant increase of amplitude in $\mathrm{HbO}$ during the stimulation and a significant decrease of amplitude in HbR during the stimulation, when compared to baseline. Here, significance was measured with the standard $z$-score between the two distributions of time points (baseline versus stimulation). This procedure compares the mean of the time points during the stimulation with the mean of the time points during baseline, considering the standard deviation of the sample during the baseline period. It is also worth noting that all analysis was performed independently for each channel, since we were also interested in analyzing spatial differences induced by the functional task across subjects.

Quantitative parameters were extracted from the HRF for posterior analysis for both $\mathrm{HbO}$ and $\mathrm{HbR}$ independently. To avoid misinterpretation of NIRS-based parameters due to noise fluctuations, we fit the HRF to a least squares moving average model with a period of five time points. Then, we quantified the magnitude of the activation as the amplitude of the $\mathrm{HRF}$ at $80 \%$ of its maximum and its latency as the time to reach $80 \%$ of its maximum amplitude. The integrated response during activation was estimated by calculating the area under the HRF, from the beginning of activation until the time it comes back to baseline.

\subsection{Statistical Analyses}

We compared the full-term and preterm groups regarding motor performance scores on Bayley-III, socioeconomic, and perinatal data employing chi-square tests (categorical/ordinal data) or independent samples $t$-tests (linear/scale data), after verifying for normality of data. Quantitative data of NIRS-based hemodynamic response (latency, magnitude of the activation, and the integrated response during activation) were expressed as the mean $(M)$ and standard deviation (SD) for the group. Using Spearman's correlation, we estimated the association between the HRF values of activated channels across the sensorimotor region and Bayley-III scores in the total sample. Statistical significance was established at 0.05 .

\section{Results}

Table 1 presents a summary of descriptive statistics for infant birth data, SES, neonatal morbidities, and motor performance, assessed by Bayley-III. There were no significant differences regarding motor performance between full-term and preterm groups (full-terms: $M=109.11, \mathrm{SD}=10.34$; preterm: $M=$ 106.04, $\mathrm{SD}=14.85 ; p=0.89)$.

Concerning the hemodynamic response measured with fNIRS, we observed robust activation in at least one channel located in the contralateral sensorimotor cortex for all infants in both groups. Across all infants, we observed a highly localized, bilateral response in the sensorimotor cortex in both groups (Fig. 2). On average, the full-term group has more channels activated, both in the contralateral and ipsilateral sensorimotor cortex than the preterm groups. The HRF in the preterm-born appears to be more localized than the functional response in the full-terms.

However, careful inspection of data showed that no infant in any group had significant activation in all the channels indicated in Fig. 2. Instead, the activation profile varied greatly in infants within the same group, as well as the total number of activated channels. In order to better understand the common activation pattern across all infants from the same group, we performed a frequency analysis considering only channels that showed activation in at least $50 \%$ of the subjects in that group. Unlike typical average responses shown in Fig. 2, the frequency analysis procedure allowed us to find the most common and repetitive response across all subjects in a group. The threshold of $50 \%$ was arbitrarily chosen so that the channels reflected robust activation in the majority of the subjects within the same group. Figures 3(a) and 3(b) shows the topographic map of the frequency analysis, with the channels color-coded by frequency 
Table 1 Demographic data and group comparisons in six-month-old full-term and preterm-born infants.

Measures

Full term

Preterm

Test

$p$

Perinatal characteristics

\begin{tabular}{|c|c|c|c|c|c|}
\hline \multicolumn{2}{|c|}{ Birth weight $(g)^{a}$} & $3,261( \pm 446)$ & $1,123( \pm 475)$ & 30.81 & $<0.001$ \\
\hline \multicolumn{2}{|c|}{ GA (weeks) ${ }^{\mathrm{a}}$} & $39( \pm 1.05)$ & $31( \pm 1.83)$ & 18.66 & $<0.001$ \\
\hline \multicolumn{2}{|c|}{ Male gender ${ }^{b}$} & $16(61 \%)$ & $8(36 \%)$ & 3.66 & 0.068 \\
\hline \multirow[t]{2}{*}{ SES $^{b}$} & $>455$ monthly & $6(24 \%)$ & $9(45 \%)$ & 0.729 & 0.460 \\
\hline & $<455$ monthly & $19(76 \%)$ & $11(55 \%)$ & & \\
\hline \multicolumn{2}{|c|}{ Days in neonatal unit ${ }^{a}$} & - & $15.60( \pm 12.11)$ & - & - \\
\hline \multicolumn{2}{|c|}{ Retinopathy of prematurity ${ }^{b}$} & - & $5(23 \%)$ & - & - \\
\hline \multicolumn{2}{|c|}{$\begin{array}{l}\text { Intraventricular hemorrhage (grade I } \\
\text { or II) }\end{array}$} & - & $16(73 \%)$ & - & - \\
\hline \multicolumn{2}{|c|}{ Bronchopulmonary dysplasia ${ }^{b}$} & - & $3(14 \%)$ & - & - \\
\hline \multicolumn{2}{|c|}{ Necrotizing enterocolitis ${ }^{b}$} & - & $1(4 \%)$ & - & - \\
\hline
\end{tabular}

Motor development measurements at six months old

\begin{tabular}{|c|c|c|c|c|}
\hline Bayley-III motor composite score ${ }^{a}$ & $109.11(10.34)$ & $106.04(14.85)$ & 0.82 & 0.89 \\
\hline $\begin{array}{l}\text { Age at motor develop. assessment } \\
\text { (days) }\end{array}$ & $205.23(2.05)$ & $195.96(2.98)$ & 2.57 & 0.13 \\
\hline Head circumference $(\mathrm{cm})^{\mathrm{a}}$ & $43.87(0.47)$ & $43.56(0.37)$ & -0.51 & 0.63 \\
\hline
\end{tabular}

${ }^{a}$ Continuous data were expressed as mean (standard deviation) and compared using independent samples $t$-test.

${ }^{\mathrm{b} C}$ Categorical data were expressed as $n(\%)$ and compared with chi-square test.

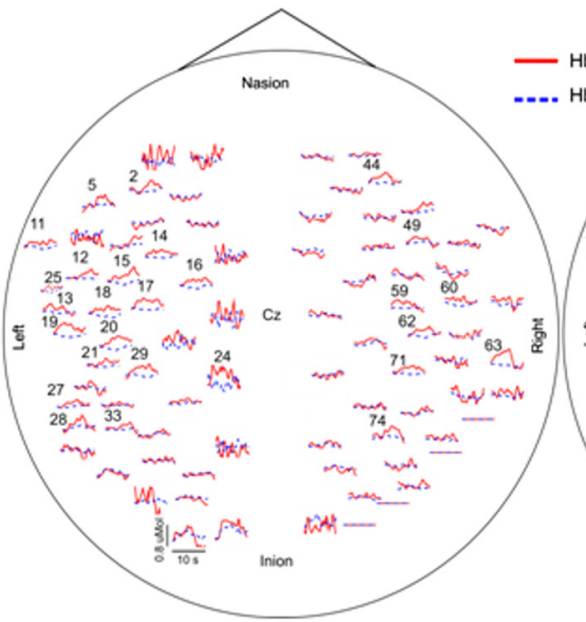

(a)

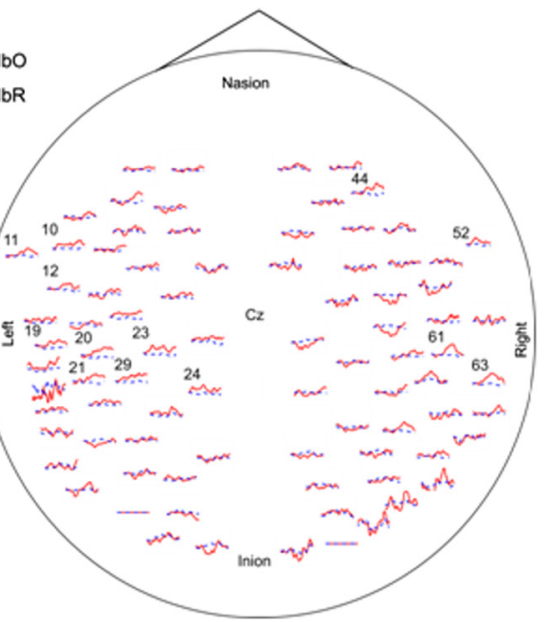

(b)

Fig. 2 Average HRF of all individual channels for all the infants in the (a) full-term and (b) preterm-born groups. The numbers next to some of the HRFs indicate the channels that show significant activation compared to baseline. They are mostly located in the sensorimotor cortex. Additional channels, located anterior and posterior to the sensorimotor cortex, also exhibited increase (decrease) in $\mathrm{HbO}(\mathrm{HbR})$ on average.

of activation in the group, for both the full-term and the preterm groups.

It is possible to note that the HRF of the full-term born group in the contralateral sensorimotor cortex is quite reproducible across infants in the group [Fig. 3(a)]. Eight different channels in this region responded to the stimulation in at least $50 \%$ of the subjects. Although full-term born infants showed activation in different channels around the ipsilateral sensorimotor cortex, the 
(a)

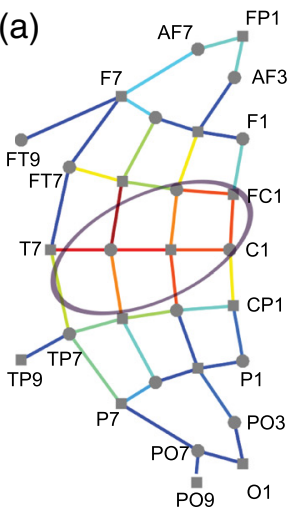

(c)

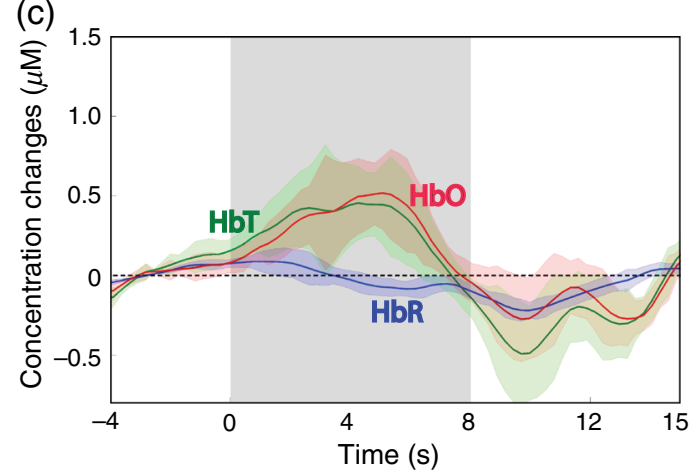

(b)

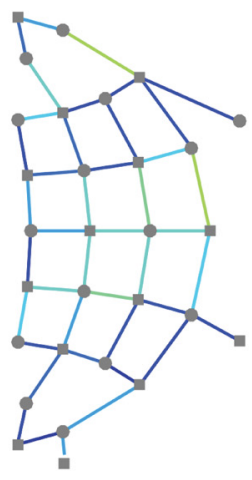

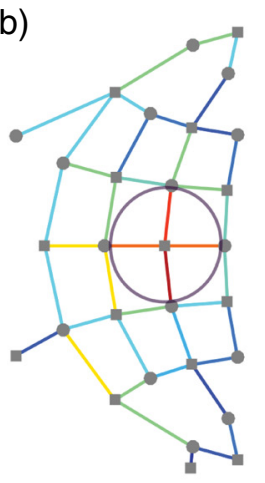
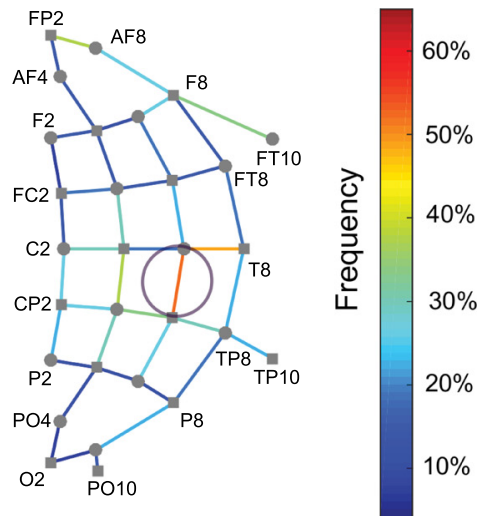

(d)

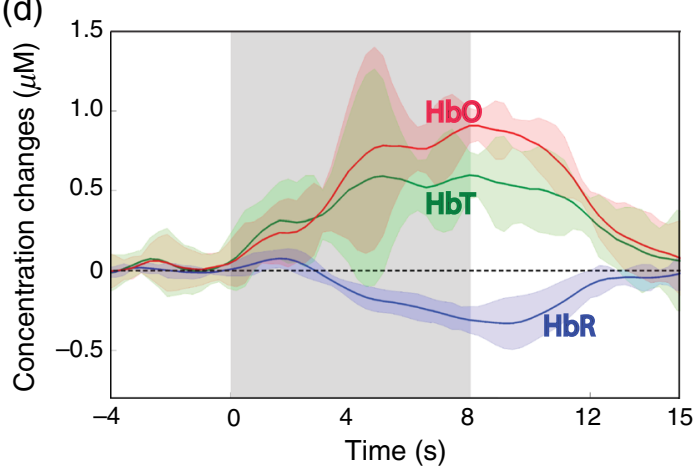

Fig. 3 Topographic map based on frequency of activation of each channel for infants in the (a) full-term and (b) preterm-born groups. The black circles in the maps identify the most frequent channels in the somatosensory cortex. No other channel in a different location reached our threshold. The average HRF across the channels that showed reproducibility (i.e., response in more than $50 \%$ of infants) in the contralateral sensorimotor cortex is plotted in (c) for the full-term born group and in (d) for the preterm-born. In the graphs, the gray area represents the stimulation period, and the dashed black line indicates the baseline level. The shadows indicate the standard error across all channels and all subjects.

ipsilateral response is quite heterogeneous; no individual channel in the ipsilateral sensorimotor cortex was activated in more than $8(31 \%)$ full-term born infants.

On the other hand, the preterm group presented a more heterogeneous activation pattern across all its subjects in the contralateral sensorimotor cortex [Fig. 3(b)]. Only four channels are frequently used in the majority of infants in the preterm group. However, in the ipsilateral sensorimotor cortex of the preterm group, the response was more reproducible, with two channels being constantly used by the infants.

Considering only the most reproducible channels in the contralateral sensorimotor cortex for each group, we observed an average (standard error) $\mathrm{HbO}$ increase of $0.51(0.03) \mu \mathrm{M}$ from baseline in full-term born, whereas the average $\mathrm{HbR}$ decrease for the same group was $-0.22(0.03) \mu \mathrm{M}$. Similarly, the average $\mathrm{HbO}$ increase from baseline across all preterm subjects was $0.71(0.04) \mu \mathrm{M}$ in the contralateral sensorimotor cortex, whereas the $\mathrm{HbR}$ decrease in the same region was $-0.33(0.02) \mu \mathrm{M}$. We did not observe any significant differences between the two groups regarding the activation's amplitude $(p=0.9$ and $p=$ 0.4 for $\mathrm{HbO}$ and $\mathrm{HbR}$, respectively).

Concerning the dynamics of the hemodynamic response, the $\mathrm{HbO}$ changes in the contralateral sensorimotor cortex had a mean (standard error) latency of $5.1(0.7) \mathrm{s}$ in the full-term born group, which is significantly faster than the response found in the preterm group, $8.2(0.9) \mathrm{s}(p=0.03)$. The time for $\mathrm{HbR}$ to reach its minimum did not vary between the groups
$(9.4 \pm 0.8 \mathrm{~s}$ and $9.8 \pm 1.1 \mathrm{~s}$ for full-term and preterm-born groups, respectively). In addition, the integrated $\mathrm{HbR}$ response (measured as the area under the curve) varied significantly between groups $(p=0.03)$. The full-term born had a mean $\mathrm{HbR}$ decrease of $4.2 \pm 1.9 \mu \mathrm{Ms}$ following motor stimulation in the contralateral hemisphere, whereas the preterm infants had a larger HbR decrease from baseline, $8.3 \pm 2.5 \mu \mathrm{Ms}$. We did not observe any significant difference between the groups in the integrated $\mathrm{HbO}$ response.

Correlation analysis was calculated between the HRF values of the reproducible activated channels in sensorimotor region [as detailed in Figs. 3(a) and 3(b)] and Bayley-III motor scores in the total sample. We identified that the HRF in the channels FC3-C3, C5-F7, and C3-CP3 was positively associated with motor performance measured by the Bayley-III (FC3-C3: Spearman's rho $=0.315, \quad p=0.020 ; \quad$ C5-F7: Spearman's rho $=0.320, p=0.022$, and C3-CP3: Spearman's rho $=$ $0.308, p=0.028$ ).

\section{Discussion}

Using an event-related design, we were able to characterize the hemodynamic response to motor stimulation in six-month-old full-term and preterm born infants with similar motor performance. From the average approach, we observed that both groups exhibited a bilateral activation response to unilateral right-hand stimulation. These results are in agreement with previous studies using different patterns of somatosensory 
stimulations in neonates (even in preterm-born at term equivalent age). ${ }^{15-18}$ However, in our study, despite both groups responding with a bilateral activation pattern, the full-term group demonstrated a more prominent unilateral contralateral response, whereas preterm infants demonstrated a more homogeneous bilateral response. In the preterm group, we observed a more reproducible ipsilateral response around C6-Cp6 and a less reproducible contralateral activated area when compared to the term group. These differences observed in full-term and preterm born lateralization of evoked sensorimotor cortex responses suggest that a considerable degree of functional lateralization may already be developed in the first months of postnatal life. This early development could explain the fact that the full-term born infants had a more homogeneous contralateral activation and only about $30 \%$ of its subjects showed bilateral activation, whereas in the preterm group, the bilateral response was more homogeneous, occurring in most subjects. Our results are consistent with the assumption that the hemispheric lateralization increases with age or development. Recent studies ${ }^{16,19}$ have identified an emerging trend of contralateral lateralization of the somatosensory systems with increased age/development and suggest that a refinement of the sensorimotor pathways, or lateralization, plays an important role during the postnatal period.

Despite consistent involvement of the sensorimotor cortex, studies produced thus far have reported conflicting results. ${ }^{19}$ However, recent studies in age-related HRF-modeling of eventrelated fMRI have improved the specificity of neonatal fMRI, ${ }^{19,20}$ demonstrating predominantly lateralized activation in the sensorimotor cortex at early ages ( 38 to 42 postconceptual weeks). ${ }^{19}$

The more homogeneous bilateral activation, observed in the majority of the infants from the preterm group, may also indicate a cortical maturity status, suggesting that the preterm cortex is less specialized than in term-born infants. Changes in location can be viewed as a direct consequence of specialization. ${ }^{21}$ One possible mechanism that converges to the specialization of the neural pathways is the selective pruning of inadequate synaptic connections and also the inhibition of alternative routes. ${ }^{22,23}$

Another possible explanation for the robust bilateral activation observed more in preterm than in full-term born infants is to consider that "the brain's resource constraints" may affect the level of cortical information processing. ${ }^{24}$ The resource constraints are a simple and inescapable consequence of the fact that the brain, like all biological systems, is subject to hard limited bioenergetic and structural resources. Therefore, preterm and full-term individuals in different rates, during the development, may have a reduced availability of resources in these regions, and additional areas will be permanently recruited. ${ }^{24}$

About the location of the cortical hemodynamic response, it is important to note that we designed the optical probe to cover the whole head. With this geometry, we were able to identify the hemodynamic response to somatosensory stimulation in sixmonth-old infants mainly localized on the sensorimotor area, despite the differences observed concerning lateralization response of each group. This activation pattern not only reinforces the adequacy of the stimulus but also rejects the occurrence of activation in unexpected cortical regions. Therefore, our optical design provided the intrinsic advantage of investigating the activation response in a broader cortical area. This kind of investigation is crucial since little is known about changes in the physiology of the neural pathways that underlies the preterm brain.
Across all subjects, we also observed a faster $\mathrm{HbO}$ response in the contralateral sensorimotor cortex in the full-term born group. Recent studies have shown a maturational trend on parameters of the hemodynamic response in terms of decreasing time-to-peak and an increasing positive peak amplitude associated with increasing age. ${ }^{25,26}$ The physiological reasons underlying these dynamic differences are probably multifactorial and involve both the development of the neural network, as many stages of the neurovascular coupling culminate in the hemodynamic changes measured by fNIRS or BOLD signals. ${ }^{25,27}$ Considering the development of neural networks, preterm birth should affect the development and maturation of cortical pathways, including cortico-cortical and thalamo-cortical connections. ${ }^{7}$ Infants born preterm, even in the absence of focal brain lesions, have reduced regional brain volumes, reduced development of both white and gray matter, ${ }^{28}$ reduced cortical surface area and gyrification, ${ }^{28,29}$ and reduced corticomotor excitability, ${ }^{30}$ which can result in slower neural conduction rates in the preterm infant brain, lengthening the mean timeto-peak response in this group.

We also observed that the increase in the average hemodynamic response in the left sensorimotor region [Figs. 3(c) and 3(d)] was correlated with higher Bayley-III motor scores. We propose that the activation response in sensorial and motor areas develops in close association with the emergence of related motor skills within the same individual. Therefore, we show that infants with the same performance in BayleyIII could have different activation during motor stimulation in the sensorial and motor regions of the cortex. Thus, fNIRS presented new and different information of which it is not clear as to the meaning of these different activation responses in long-term or in clinical context. Moreover, previous studies have shown the association between neurodevelopmental outcomes and brain status. ${ }^{31,32}$ Using diffusion tensor imaging, Yeo et al. ${ }^{33}$ showed that the maturation status of motor pathways appears to be related to different periods of fine and gross motor development. Conventional MRI data, volumetric measurements, brain metrics, and surface-based studies of cerebral cortex have shown moderate to high predictive value for neurodevelopment, such as motor performance, cerebral palsy, and sensory impairments (see Ref. 34 for a review). Therefore, an understanding correlation of neurobiology and behavior in preterm infants requires further efforts to clarify the individual and combined contributions of multiple risk factors, inherent to premature birth to neurodevelopmental outcomes. Assessments of brain status and neuropsychological skills are likely to play a critical role in decoding these mechanisms. ${ }^{34}$

Even in infants with similar mean motor scores (using Bayley-III Scale), we observed differences in brain activation between groups. We questioned whether this is due to a fragility of the scale for specific populations or whether these differences in hemodynamics may suggest a compensatory mechanism to maintain function. Bayley-III has been previously criticized for the possibility of developmental status overestimates and presents a poor predictor of later functioning. ${ }^{35}$

It was not our intention to establish a causal relationship between motor development and cortical activity during motor stimulation because we understand that the relation between neurobiology and behavior should be much more complex than the direct correlation between brain activity and behavioral response. ${ }^{36}$ The modest values of association between the two instruments (fNIRS and Bayley-III) in this study reinforce this 
view. Furthermore, our limited sample size did not allow us to stratify the preterm group neither by GA nor by motor development outcome (i.e., normal or delayed in motor domain). This may be seen as a limitation, but we reinforce that we exclude many risk factors for motor changes that are often associated with prematurity and their complications.

In conclusion, our results suggest that full-term born infants at six months of age have different task-induced activation response in motor cortex compared with preterm infants with similar motor performance at Bayley-III Scale, both in relation to the activated area and to the magnitude and dynamic aspects of the activation response. The brain hemodynamic response measured by NIRS appears to be related, at least to some degree, to the motor performance exhibited by these infants. The results support the need for additional efforts to better understand the mechanisms involved on early development of motor abilities in premature infants and suggest that fNIRS can become a suitable auxiliary tool to investigate aspects of neural basis on early development of motor abilities.

\section{Disclosures}

The authors have no conflicts of interest to declare.

\section{Acknowledgments}

This work was funded by the Minas Gerais Research Foundation-FAPEMIG (Grant No. 21550) and the São Paulo Research Foundation-FAPESP (Grant Nos. 2012/ 02500-8 and 2013/07559-3).

\section{References}

1. K. Velikos et al., "Bayley-III scales at 12 months of corrected age in preterm infants: patterns of developmental performance and correlations to environmental and biological influences," Res. Dev. Disabil. 45, 110119 (2015).

2. T. A. Goyen and K. Lui, "Longitudinal motor development of "apparently normal' high-risk infants at 18 months, 3 and 5 years," Early Hum. Dev. 70, 103-115 (2002).

3. M. N. Spann et al., "Morphological features of the neonatal brain support development of subsequent cognitive, language, and motor abilities," Hum. Brain Mapp. 35, 4459-4474 (2014).

4. R. S. Moreira et al., "Factors influencing the motor development of prematurely born school-aged children in Brazil," Res. Dev. Disabil. 35, 1941-1951 (2014).

5. L. C. H. Fernald et al., Examining Early Child Development in LowIncome Countries, The World Bank, Washington, DC (2009).

6. J. A. Eyre, "Developmental plasticity of the corticospinal system," Chapter 3 in Plasticity in the Human Brain: Investigations with Transcranial Magnetic Brain Stimulation, S. Boniface and U. Ziemann, Eds., pp. 62-89, Cambridge University Press, Cambridge (2003).

7. C. D. Smyser et al., "Longitudinal analysis of neural network development in preterm infants," Cereb. Cortex 20, 2852-2862 (2010).

8. D. A. Boas et al., "Twenty years of functional near-infrared spectroscopy: introduction for the special issue," Neurolmage 85, 1-5 (2014).

9. S. Lloyd-Fox, A. Blasi, and C. E. Elwell, "Illuminating the developing brain: the past, present and future of functional near infrared spectroscopy," Neurosci. Biobehav. Rev. 34, 269-284 (2010).

10. Associação Brasileira de Empresas de Pesquisa (ABEP), Critério de Classificação Econômica Brasil, 2013, http://www.abep.org/Servicos/ Download.aspx?id=01 (24 April 2006).

11. N. Bayley, Bayley Scales of Infant Development, 3rd ed., Administration Manual, Harcourt Assessment, San Antonio, Texas (2009).

12. G. H. Klem et al., "The ten-twenty electrode system of the international federation," Electroencephalogr. Clin. Neurophysiol. 52, 3-6 (1999).

13. T. J. Huppert et al., "HomER: a review of time-series analysis methods for near-infrared spectroscopy of the brain," Appl. Opt. 48, D280-D298 (2009).
14. A. Lima and J. Bakker, "Near-infrared spectroscopy for monitoring peripheral tissue perfusion in critically ill patients," Rev. Bras. Ter. Intensiva 23, 341-351 (2011).

15. A. G. Allievi et al., "Maturation of sensori-motor functional responses in the preterm brain," Cereb. Cortex 26, 402-413 (2015).

16. S. G. Erberich et al., "Somatosensory lateralization in the newborn brain," NeuroImage 29, 155-161 (2006).

17. S. G. Erberich et al., "Functional MRI in neonates using neonatal head coil and MR compatible incubator," Neurolmage 20, 683-692 (2003).

18. T. Arichi et al., "Somatosensory cortical activation identified by functional MRI inpreterm and term infants," NeuroImage 49, 2063-2071 (2010).

19. L. Scheef et al. "Functional laterality of task-evoked activation in sensorimotor cortex of preterm infants: an optimized $3 \mathrm{~T}$ fMRI study employing a customized neonatal head coil," PLoS One 12, e0169392 (2017).

20. R. Cusack et al., "Optimizing stimulation and analysis protocols for neonatal fMRI," PLoS One 10, e0120202 (2015).

21. M. H. Johnson, "Functional brain development in infants: elements of an interactive specialization framework," Child Dev. 71, 75-81 (2000).

22. R. A. Jacobs, "Computational studies of the development of functionally specialized neural modules," Trends Cognit. Sci. 3, 31-38 (1999).

23. J. Shrager and M. H. Johnson, "Dynamic plasticity influences the emergence of function in a simple cortical array," Neural Networks 9, 1119_ 1129 (1996).

24. M. A. Just and S. Varma, "The organization of thinking: what functional brain imaging reveals about the neuroarchitecture of complex cognition," Cognit. Affect Behav. Neurosci. 7, 153-191 (2007).

25. T. Arichi et al., "Development of BOLD signal hemodynamic responses in the human brain," NeuroImage 63, 663-673 (2012).

26. M. T. Colonnese et al., "Development of hemodynamic responses and functional connectivity in rat somatosensory cortex," Nat. Neurosci. 11, 72-79 (2008)

27. J. J. Harris, C. Reynell, and D. Attwell, "The physiology of developmental changes in BOLD functional imaging signals," Dev. Cognit. Neurosci. 1, 199-216 (2011).

28. J. F. de Kieviet et al., "Brain development of very preterm and very low birth-weight children in childhood and adolescence: a meta-analysis," Dev. Med. Child Neurol. 54, 313-323 (2012).

29. M. Ajayi-Obe et al., "Reduced development of cerebral cortex in extremely preterm infants," Lancet 356, 1162-1163 (2000).

30. J. B. Pitcher et al., "Reduced corticomotor excitability and motor skills development in children born preterm," J. Physiol. 590, 5827-5844 (2012).

31. S. J. Counsell et al., "Specific relations between neurodevelopmental abilities and white matter microstructure in children born preterm," Brain 131(12), 3201-3208 (2008).

32. K. J. Rademaker et al., "Larger corpus callosum size with better motor performance in prematurely born children," Semin. Perinatol. 28, 279_ 287 (2004).

33. S. S. Yeo, S. H. Jang, and S. M. Son, "The different maturation of the corticospinal tract and corticoreticular pathway in normal brain development: diffusion tensor imaging study," Front. Hum. Neurosci. 8, 573 (2014).

34. A. M. Mathur, J. J. Neil, and T. E. Inder, "Understanding brain injury and neurodevelopmental disabilities in the preterm infant: the evolving role of advanced magnetic resonance imaging," Semin. Perinatol. 34, 57-66 (2010)

35. P. J. Anderson and A. Burnett, "Assessing developmental delay in early childhood-concerns with the Bayley-III scales," Clin. Neuropsychol. 31(2), 371-381 (2017).

36. S. J. Paterson et al., "Development of structure and function in the infant brain: implications for cognition, language and social behavior," Neurosci. Biobehav. Rev. 30(8), 1087-1105 (2006).

Suelen Rosa de Oliveira received her MS degree in nursing from the School of Nursing at the Federal University of Minas Gerais in 2009 and her PhD in health sciences/child health from the Faculty of Medicine at the Federal University of Minas Gerais in 2016, studying the association between hemodynamic response and motor development in preterm and full-term born infants at the first year of life. 
Ana Carolina Cabral de Paula Machado is a registered physical therapist and a specialist in intensive therapy in neonatology and pediatrics by the Federal Council of Physical Therapy and Occupational Therapy. He is a master and doctoral student in health sciences (child and adolescent health) at UFMG.

Lívia de Castro Magalhães is a registered occupational therapist, $\mathrm{PhD}$ in education, and a professor of the Graduate Program in Rehabilitation Science, UFMG. She is a subcoordinator of the folow-up program for preterm neonates at UFMG's Hospital.

Sergio L. Novi is a PhD candidate in the Laboratory of Biomedical Optics, University of Campinas, Brazil. His research focus on developing methods for functional connectivity involving multimodal neuroimaging with NIRS. He has contributed to the translation of graph theory to investigate NIRS data. Over the last years, he has also contributed on applying novel time-series methods for functional NIRS in a variety of problems.
Rickson C. Mesquita is an assistant professor of physics at the University of Campinas (UNICAMP, São Paulo, Brazil). He leads a group in biomedical optics with interests focused on designing new instrumentation and developing innovative methods for diffuse optics in biological tissue. He has extensive experience in translating diffuse optical techniques for clinical applications.

Débora Marques de Miranda is an associate professor of pediatrics at UFMG. She received her MD and PhD degrees. She is a coordinator of Imaging Center at UFMG.

Maria Cândida Ferrarez Bouzada received her $\mathrm{PhD}$ in child health. She is a pediatrician, neonatologist and a professor of the Graduate Program in pediatrics-UFMG. She is a member of the folow-up program for preterm neonates at UFMG Clinical Hospital and Consultant of Ministery of Health for Kangaroo Method.

Biographies for the other authors are not available. 